\title{
Use of Community Health Workers and Patient Navigators to Improve Cancer Outcomes Among Patients Served by Federally Qualified Health Centers: A Systematic Literature Review
}

Katherine B. Roland, ${ }^{1, * *}$ Erin L. Milliken, Elizabeth A. Rohan, Amy DeGroff, Susan White, Stephanie Melillo, William E. Rorie, Carmita-Anita C. Signes, and Paul A. Young ${ }^{2}$

\begin{abstract}
Introduction: In the United States, disparities in cancer screening, morbidity, and mortality are well documented, and often are related to race/ethnicity and socioeconomic indicators including income, education, and healthcare access. Public health approaches that address social determinants of health have the greatest potential public health benefit, and can positively impact health disparities. As public health interventions, community health workers (CHWs), and patient navigators (PNs) work to address disparities and improve cancer outcomes through education, connecting patients to and navigating them through the healthcare system, supporting patient adherence to screening and diagnostic services, and providing social support and linkages to financial and community resources. Clinical settings, such as federally qualified health centers (FQHCs) are mandated to provide care to medically underserved communities, and thus are also valuable in the effort to address health disparities. We conducted a systematic literature review to identify studies of cancer-related CHW/PN interventions in FQHCs, and to describe the components and characteristics of those interventions in order to guide future intervention development and evaluation.

Method: We searched five databases for peer-reviewed CHW/PN intervention studies conducted in partnership with FQHCs with a focus on cancer, carried out in the United States, and published in English between January 1990 and December 2013.

Results: We identified 24 articles, all reporting positive outcomes of CHW/PNs interventions in FQHCs. CHW/PN interventions most commonly promoted breast, cervical, or colorectal cancer screening and/or referral for diagnostic resolution. Studies were supported largely through federal funding. Partnerships with academic institutions and community-based organizations provided support and helped develop capacity among FQHC clinic leadership and community members.

Discussion: Both the FQHC system and CHW/PNs were borne from the need to address persistent, complex health disparities among medically underserved communities. Our findings support the effectiveness of CHW/PN programs to improve completion and timeliness of breast, cervical, and colorectal cancer screening in FQHCs, and highlight intervention components useful to design and sustainability.
\end{abstract}

Keywords: cancer prevention; cancer screening; chronic disease; community health worker; FQHC; medically underserved; patient navigation

\footnotetext{
${ }^{1}$ Division of Cancer Prevention and Control, Centers for Disease Control and Prevention, Atlanta, Georgia.

${ }^{2}$ NOVA Research Company, Silver Spring, Maryland.

Current affiliation: Division of HIV/AIDS Prevention, Centers for Disease Control and Prevention, Atlanta, Georgia.

*Address correspondence to: Katherine B. Roland, MPH, Division of HIV/AIDS Prevention, Centers for Disease Control and Prevention, 1600 Clifton Road, E-37, Atlanta, GA 30329, E-mail: kroland@cdc.gov

(c) Katherine B. Roland et al. 2017; Published by Mary Ann Liebert, Inc. This is an Open Access article distributed under the terms of the Creative Commons Attribution License, which permits unrestricted use, distribution, and reproduction in any medium, provided the original work is properly cited. Mary Ann Liebert, Inc. offers reprint services for those who want to order professionally produced copies of articles published under the Creative Commons Attribution (CC BY) license. To obtain a price quote, email Reprints@liebertpub.com. Please include the article's title or DOI, quantity, and delivery destination in your email.
} 


\section{Introduction}

In the United States, disparities in cancer screening, diagnosis, time to treatment, and morbidity and mortality are well documented, and often are related to an individual's race/ethnicity, income, education, and healthcare access. ${ }^{1,2}$ To address health disparities, public health approaches that consider the physical, social, cultural, organizational, community, economic, legal, or policy contexts within which people live have the greatest potential benefit. ${ }^{3-5}$ Specifically, increasing access to quality healthcare for the medically underserved and increasing the capacity of the prevention workforce are key recommendations to effectively address health disparities and achieve health equity. ${ }^{4,6-8}$

Community health workers (CHWs) and patient navigators (PNs) are members of the prevention workforce who increase access to care for the medically underserved. $^{8-10} \mathrm{~A} \mathrm{CHW}$ is a nonclinical, frontline, public health worker who is a trusted member and/or has an unusually close understanding of the community served, and can function to bridge the gap between an individual and the healthcare system. ${ }^{11} \mathrm{CHWs}$ are also commonly referred to as promotores de salud, community health representatives, community health advisors, lay health educators, and lay health advisors, among other titles. ${ }^{12}$ The PN role was originally developed in the early 1990s to address cancer disparities by reducing barriers to timely breast cancer diagnosis and treatment among poor women in Harlem. Since that time, the scope of PNs has expanded across several cancers and the entire cancer spectrum from prevention, detection, diagnosis, treatment, and survivorship to the end of life. ${ }^{13}$ Today, both CHWs and PNs work to address health disparities across many chronic diseases, and improve health by providing education and advocacy services, addressing individuals' barriers to care, and linking and navigating patients through the healthcare system, and to financial and community resources. CHW/PN efforts have led to increases in cancer screening and timely completion of diagnostic follow-up and cancer treatment initiation. ${ }^{14-16}$

Federally qualified health centers (FQHCs) are legislatively mandated to provide primary care for medically underserved communities, and thus are ideal settings for CHW/PN interventions. The U.S. Department of Health and Human Services' Health Resources and Services Administration (HRSA) currently funds $\sim 1300$ FQHCs serving over 22 million patients annually. ${ }^{17} \mathrm{FQHCs}$ are critical to addressing cancer disparities among the med- ically underserved in the United States, ${ }^{18,19}$ as FQHC patients often are at greater risk for developing cancer and experiencing worse cancer outcomes compared with the general U.S. population. ${ }^{2}$ The Affordable Care Act (ACA) established the Community Health Center Fund, providing \$11 billion over a 5-year period (2011-2015) for the operation, expansion, and construction of health centers. ${ }^{20,21}$ Consequently, FQHCs as safety-net healthcare organizations have been increasingly responsible for providing crucial primary care services for vulnerable populations. ${ }^{22}$ Beyond expanding FQHCs, ACA requires that most insurance plans provide certain in-network preventive health services, including breast, cervical, and colorectal cancer screening, at no cost to patients when obtained in-network. CHW/PN programs can facilitate prevention activities and can help the newly insured and others gain access to primary care and complete cancer screening. ${ }^{19}$

$\mathrm{CHW} / \mathrm{PN}$ programs and FQHCs are natural allies. However, little has been systematically documented about the effectiveness of $\mathrm{CHW} / \mathrm{PN}$ programs conducted in FQHCs for cancer prevention and control. Therefore, we conducted a systematic review of the peer-reviewed literature to identify studies of cancerrelated CHW/PN interventions conducted in FQHCs and other HRSA-funded community health centers, and FQHC Look-Alike clinics. FQHC Look-Alikes share a mission with FQHCs to provide primary care to medically underserved communities and are eligible for FQHC reimbursement structures and discounted drug pricing, but are not funded by HRSA and therefore cannot be called FQHCs (https://bphc.hrsa.gov/ programopportunities/lookalike). Our objectives were to first identify studies that evaluated the effectiveness of these interventions in addressing cancer prevention and control disparities among the medically underserved (e.g., increasing screening, reducing time to diagnosis and treatment, reducing barriers to care) and to subsequently describe the components and characteristics of those interventions to guide future intervention development and evaluation.

\section{Methods}

In January 2014, we searched five databases: PubMed/ Medline, Scopus, Web of Science, CINAHL (Cumulative Index to Nursing and Allied Health Literature), and PsychINFO. Search terms utilized were based upon previous research ${ }^{19,23-25}$ and organized into three categories: (1) position/role, (2) clinical setting, and (3) disease focus (Table 1). We limited the search 
Table 1. Search Terms Used to Identify Relevant Literature

Position/role Adherence supporter*; Camp health aide*; Client navigat*; Community health advis*; Community health advocate*;

Community health aide*; Community health promoter*; Community health representative*; Community health worker*; Community navigator*; Community outreach worker*; Consejer*; Embajador*; Health aide*; Health coach*; Health communicator*; Health guide*; Health navigat*; Health volunteer*; Lay health adviser*; Lay health advisor*; Lay health advocate*; Lay health educator*; Lay health promoter*; Lay health representative*; Lay health worker*; Lay navigat*; Lay outreach worker*; Native American navigat*; Navigat*; Navigation program*; Navigation service*; Navigator nurse*; Nurse navigat*; Outreach worker*; Patient navigat*; Peer counselor*; Peer educator*; Peer health advisor*; Peer health counselor*; Peer health educator*; Peer health promoter*; Peer leader*; Peer navigator*; Promotor*

Clinical setting Community health center*, CHC*; Community health clinic*; Federally Qualified Health Center*, FQHC*; FQHC Look-Alike*; Neighborhood health center*; Neighborhood health clinic*; Tribal health center*; Tribal health clinic*; Indian Health Service

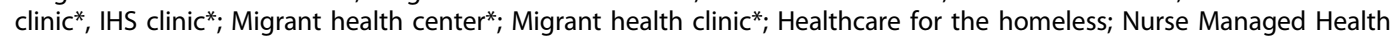
Clinic*, NMHC*; Nurse managed health center*; Public health clinic*; Urban health center*; Rural health center*; Urban health clinic*; Rural health clinic*

Disease focus Cancer; Neoplasms

*Indicates use of wildcard.

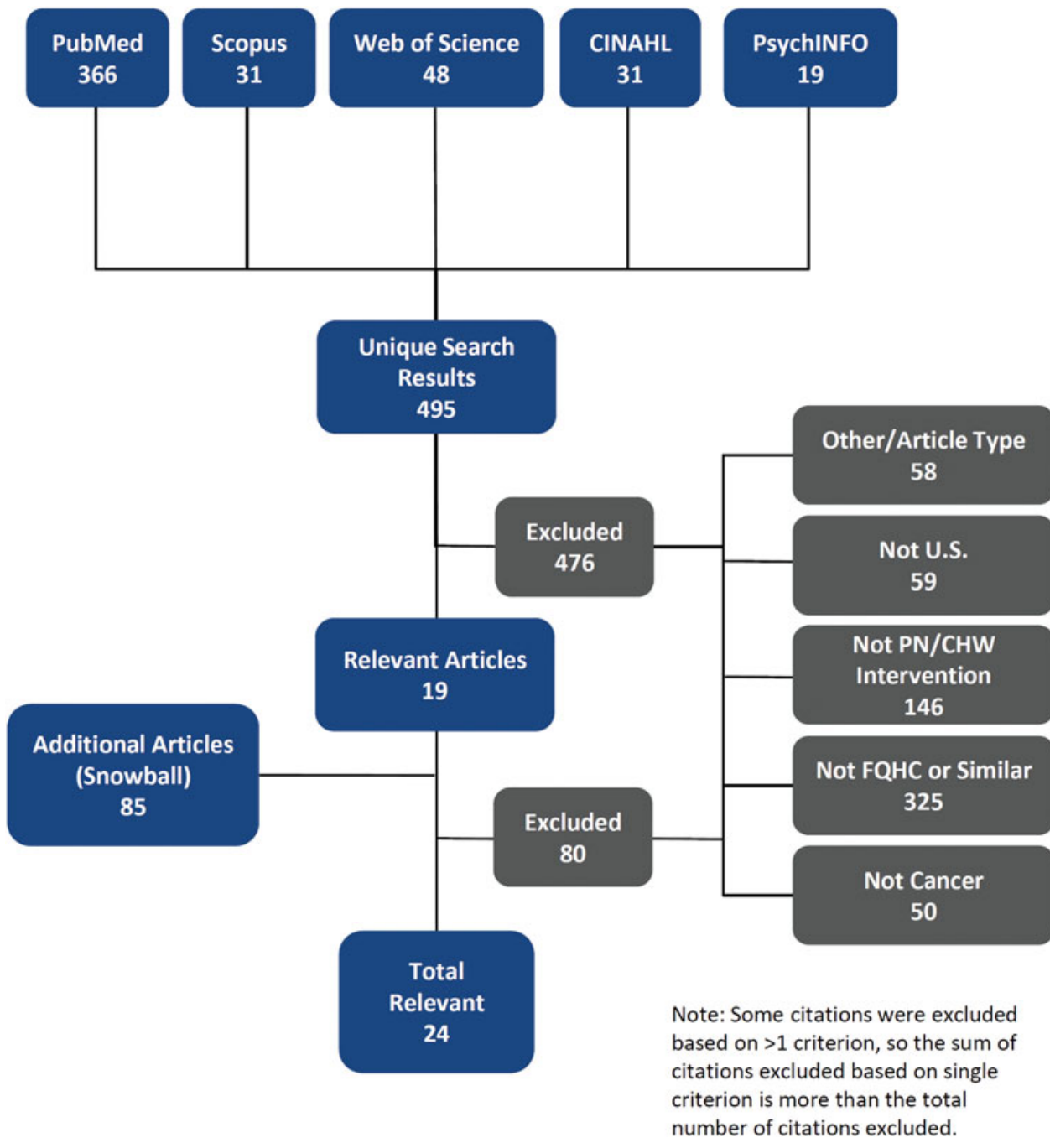

FIG. 1. Flowchart illustrating relevancy literature review selection process. 


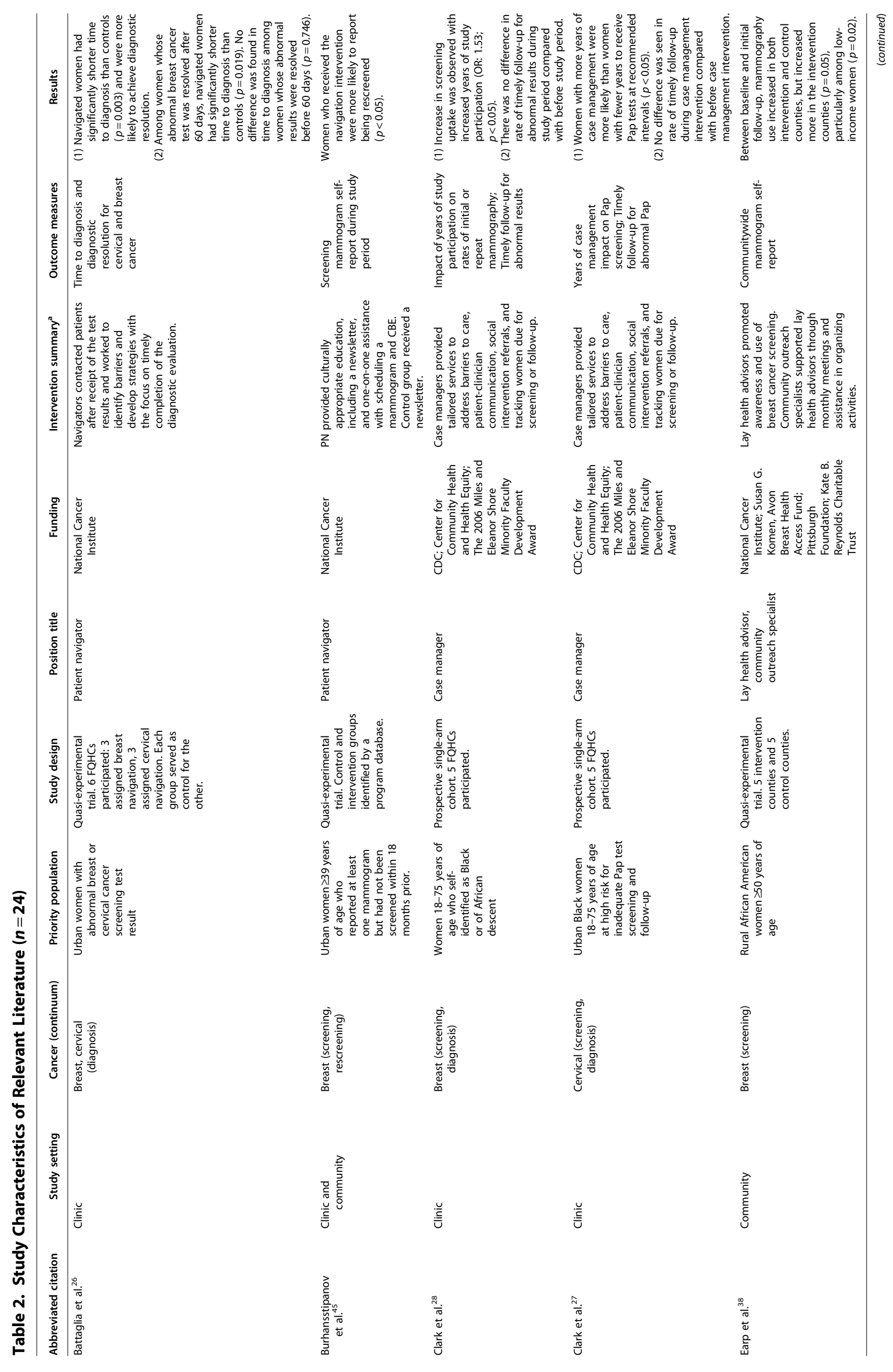




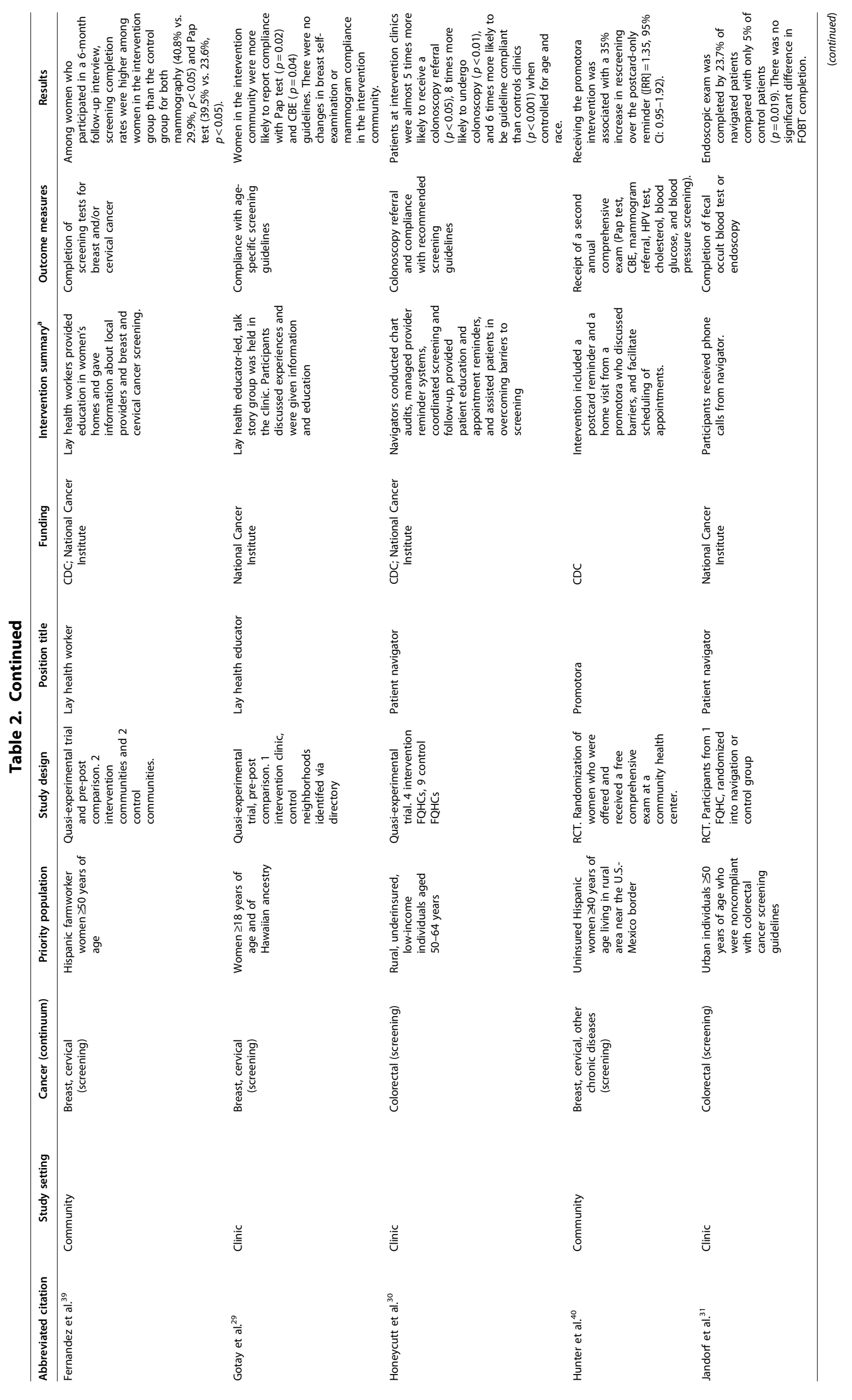




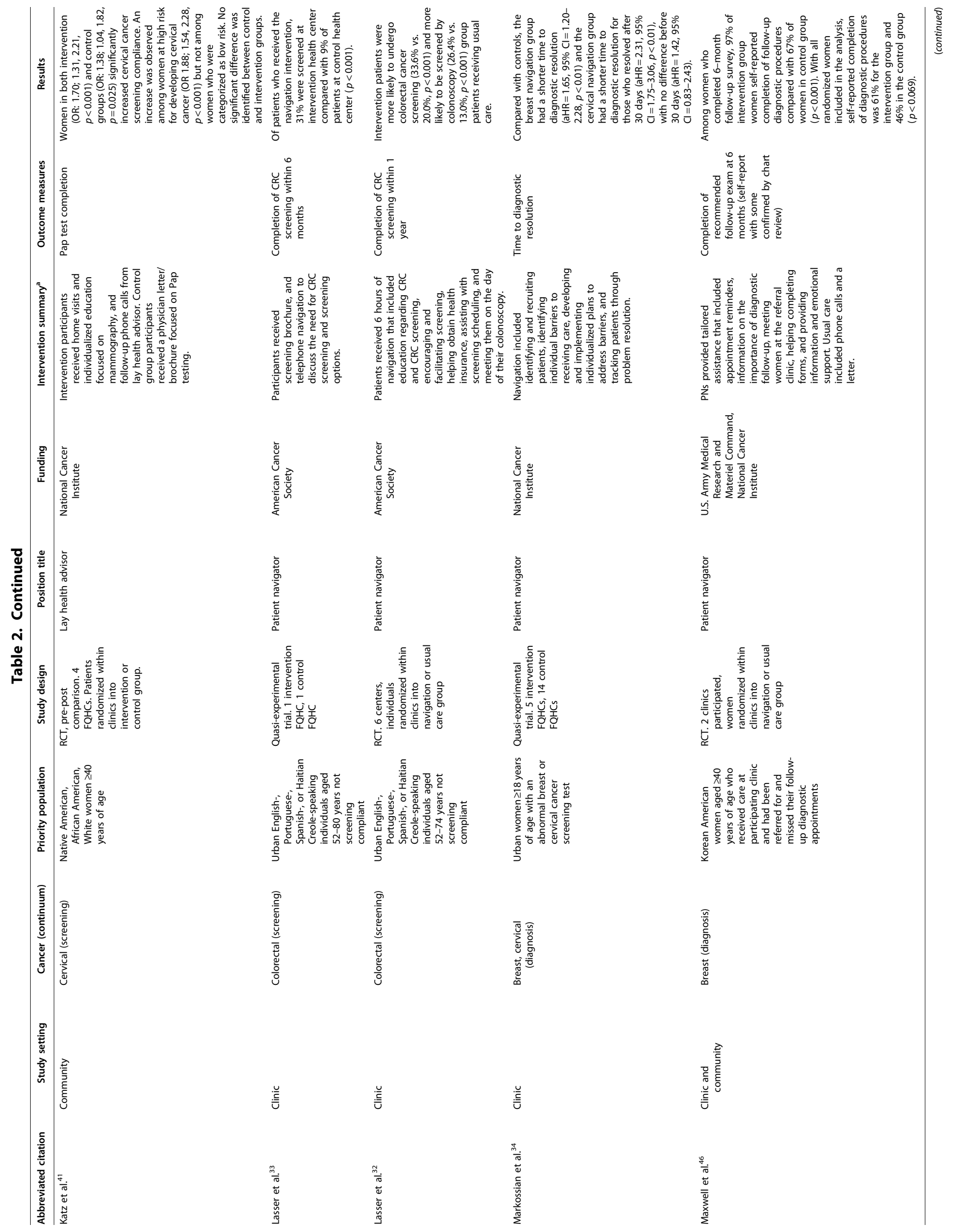




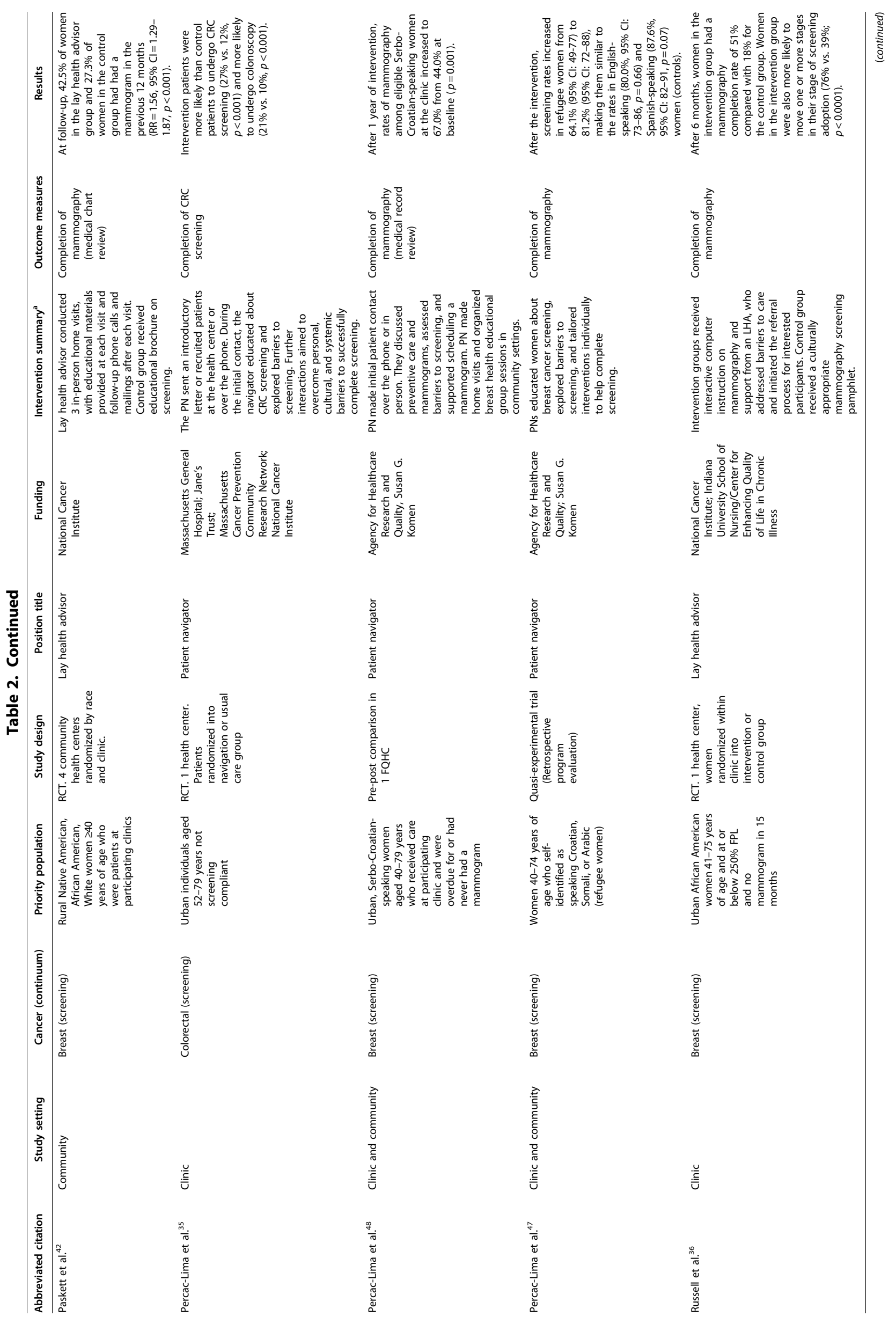




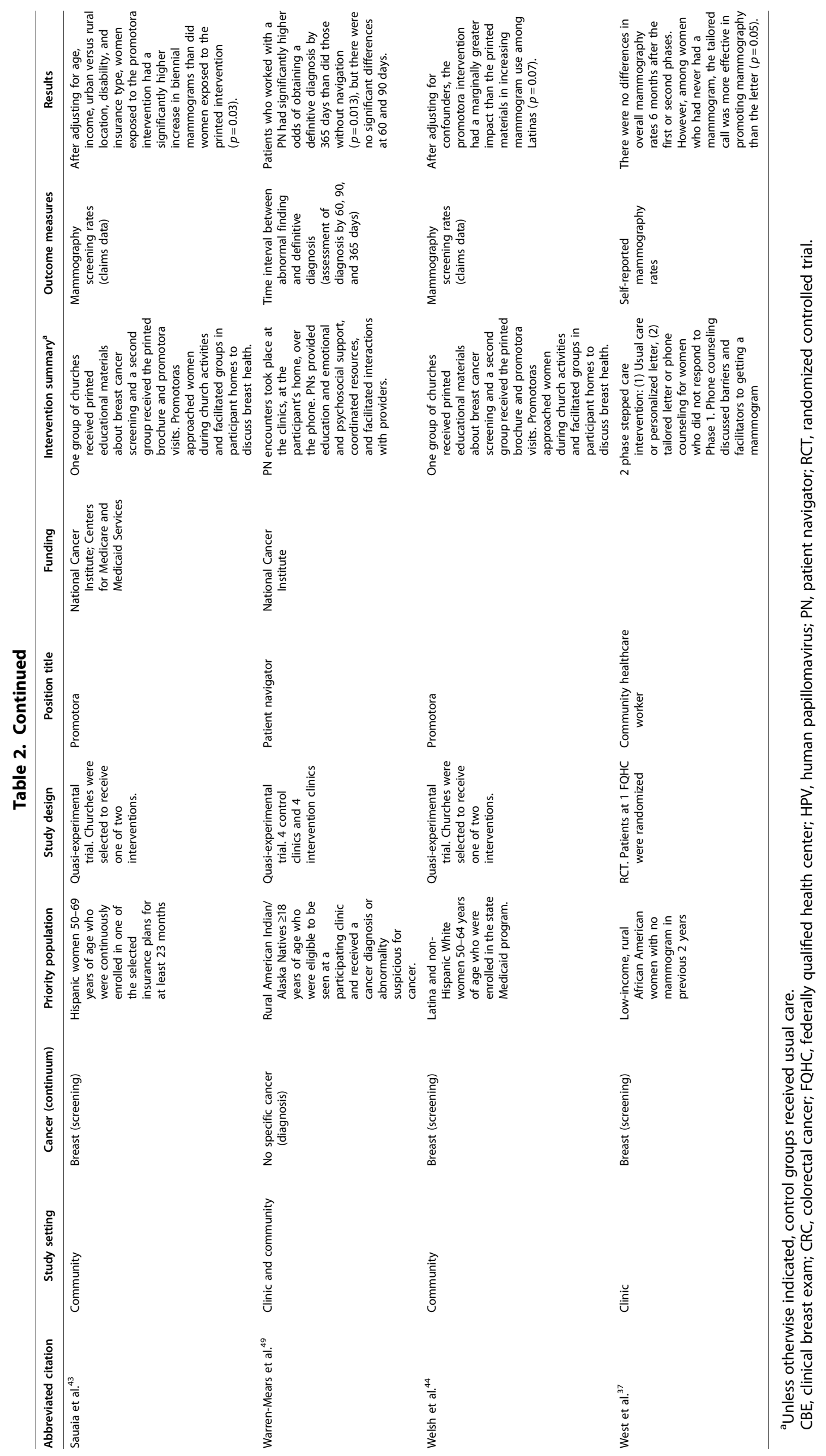


to articles published in English between January 1990 and December 2013.

A total of 495 articles were identified. Upon initial review, 58 articles were excluded because they were not original research (e.g., general descriptions of patient navigation, commentaries, editorials, book reviews, systematic literature reviews). Two coauthors independently reviewed the remaining articles (abstract and/or full-text), applying the following additional inclusion criteria: (1) U.S. study of CHW/PN intervention with results, (2) conducted in an FQHC or in partnership with an FQHC (including HRSAfunded nurse managed health clinics and community health centers, FQHC Look-Alikes and other health centers that do not receive HRSA funding but provide comprehensive primary healthcare services to medically underserved populations, and Indian Health Service clinics), and (3) focused on cancer. Discrepancies in assessment between the two reviewers were resolved through discussion, instead of a third reviewer. The two reviewers were subject matter experts and principle investigators of the study, and therefore best suited to discuss nuance of an individual article and come to a consensus of its relevancy. An objective third reviewer may not have had the subject matter expertise or the intimate understanding of the study to make a clear judgement about the relevancy of an article.

Of the 495 articles identified by the original searches, 19 met all inclusion criteria. A second tier search examined the bibliographies of the 19 identified articles, along with the bibliographies of published systematic literature review articles identified in the original search (excluded because they were not original research studies). From this secondary search, 85 additional potentially relevant articles were identified and reviewed by the two reviewers using the criteria noted above. Of these, five met the inclusion criteria (Fig. 1). In total, we identified 24 articles as relevant to this review.

Data from relevant articles were abstracted by the two coauthors. Abstracted data included study details (design, data collection method, outcomes and results, and funding source); cancer focus; study setting (HRSA affiliation of clinic, clinic type, clinic name, and number of clinics involved in study); $\mathrm{CHW} / \mathrm{PN}$ position information (role/title, education/training, whether paid or volunteer, whether full-time or part-time, funding source for position, whether demographically matched to target population, goals for the position, activities conducted, location of activities, method of communi- cation with client, interaction with data systems, supervision, level of integration into medical team); broader intervention information (collaboration or partnerships, program barriers and facilitators, and relevant program policies); and intervention target population information (gender, age, race/ethnicity, rural/urban). Discrepancies in abstracted data were discussed until resolution was achieved.

\section{Results}

\section{Study characteristics}

The 24 articles included in this review are presented in Table 2. These articles profile $\mathrm{CHW} / \mathrm{PN}$ interventions conducted in the clinical setting of $\mathrm{FQHCs},{ }^{26-37}$ in the community through partnerships with FQHCs, ${ }^{38-44}$ and in both the clinic and community settings. ${ }^{45-49}$ Interventions targeted cancer screening ${ }^{27-33,35-45,47,48}$ and diagnosis ${ }^{26-28,34,46,49}$ for breast, ${ }^{26,28,29,34,36-40,42-48}$ cervi$\mathrm{cal}^{26,27,29,34,39-41}$ or colorectal cancer. ${ }^{30-33,35}$ One article focused on cancer, but did not specify the type of cancer for the intervention activities. ${ }^{49}$ Interventions focused on medically underserved populations, including Croatian-, Somali-, Arabic-, English-, Portuguese-, Spanish-, and Haitian Creole-speaking ${ }^{32,33,47}$; African American, ${ }^{27,38,41,42}$ Hispanic, ${ }^{39,40,43}$ Native American, ${ }^{41,42}$ and Native Hawaiian, ${ }^{29}$ in large urban cities, ${ }^{26,27,31-36,43-48}$ rural counties in the South, ${ }^{30,37,38,41,42}$ a suburban neighborhood in Hawaii, ${ }^{29}$ the U.S.-Mexico border region, ${ }^{39,40}$ and Northwest Pacific Tribal clinics. ${ }^{49}$

The studies included in this review represented a variety of study designs. Most studies were either randomized controlled trials (RCTs; $n=9)^{31,32,35-37,40-42,46}$ or quasiexperimental designs $(n=12){ }^{26,29,30,33,34,38,39,43-45,47,49}$ Two studies utilized a single-arm cohort design, ${ }^{27,28}$ and one study utilized a pre- and postintervention comparison. ${ }^{48}$

The literature referred to $\mathrm{CHW} / \mathrm{PNs}$ using numerous titles, reflecting their widely variable roles and responsibilities, including $\mathrm{PN}^{26,30-35,45-49}$ lay health advisor, ${ }^{36,38,41,42}$ promotora, ${ }^{40,43,44}$ case manager, ${ }^{27,28}$ community healthcare worker, ${ }^{37}$ community outreach specialist, ${ }^{38}$ lay health educator, ${ }^{29}$ and lay health worker. ${ }^{39}$ The title PN was used most commonly in articles profiling clinic-based interventions, while the titles lay health advisor and promotora were more common to articles describing community-based interventions. This article does not distinguish between titles when presenting study results below.

Partnerships are often recognized as essential in the development and implementation of $\mathrm{CHW} / \mathrm{PN}$ 
programs. Some studies included in this review partnered with CDC's National Breast and Cervical Cancer Early Detection Program to facilitate patients' referral to screening services. ${ }^{34,37-39,44,46}$ Other studies involved partnerships with a Community Advisory Panel to monitor and facilitate the community-based research process, ${ }^{26}$ academic researchers, and community members to develop study methods and conduct analysis, ${ }^{29,37}$ churches, the state department of public health, and the health center to host the sites of intervention activities, ${ }^{44}$ and a nonprofit organization to cover costs for navigators and colonoscopies. ${ }^{30}$ One intervention study was conducted by a coalition of community activists, public health officials, academic researchers, and the Boston Public Health Commis$\operatorname{sion}^{27,28}$ (data not included in a table).

Of the 24 relevant articles, 22 acknowledged funding from at least one federal agency. The most common federal funding source was the National Cancer Institute, ${ }^{26,29-31,34-39,41-46,49}$ followed by CDC, ${ }^{27,28,30,37,39,40}$ Center for Medicaid and Medicare Services, ${ }^{43,44}$ the Agency for Healthcare Research and Quality, ${ }^{47,48}$ and the U.S. Army Medical Research and Materiel Command. ${ }^{46}$ Several articles also cited funding from nonprofit organizations, including the American Cancer Society, ${ }^{32,33}$ Susan G. Komen, ${ }^{38,47,48}$ Kate B. Reynolds Charitable Trust, ${ }^{38}$ Pittsburgh Foundation, ${ }^{38}$ Avon Breast Health Access Fund, ${ }^{38}$ and Jane's Trust. ${ }^{35}$ Other funding sources included the Massachusetts Cancer Prevention Community Research Network ${ }^{35}$ and other institutional support. ${ }^{28,35,36}$

\section{Outcome measures}

Reported cancer-related outcome measures for relevant studies included completion of screening, ${ }^{27-29,31-33,35-}$ $39,41-45,47-49$ followed by time to diagnosis, and completion of diagnostic resolution, ${ }^{26-28,34,46,49}$ and referral for screening by a CHW/PN. ${ }^{30}$ One study measured receipt of a follow-up wellness exam as a study outcome (which included breast and cervical cancer screening, blood pressure screening, and blood glucose testing). ${ }^{40}$

\section{Study results}

All but two studies reported statistically significant positive outcomes from the $\mathrm{CHW} / \mathrm{PN}$ interventions $(p \leq 0.05)$. One RCT that found a $35 \%$ increase in rescreening in the intervention group but reported nonsignificant confidence intervals $(\mathrm{RR}=1.35,95 \%$ CI: 0.95-1.92), ${ }^{40}$ and one quasi-experimental trial identified increased mammography rates in the interven- tion group, but the increases were not significant when compared to controls $(p \geq 0.07) .{ }^{47}$ While all studies were conducted among subpopulations that may experience cancer disparities or barriers to care, some studies specifically targeted under-screened or never screened individuals, or those with abnormal cancer screening tests.

Studies that did not specify additional risk factors in the study population $(n=11$; prospective single-arm cohort, ${ }^{28}$ quasi-experimental trials, ${ }^{29,30,38,39,43,44,47}$ $\mathrm{RCT}^{40-42}$ ) reported increases in mammography, ${ }^{28,29,38,39,42-44,47}$ Pap tests, ${ }^{29,39,41}$ referral to colonoscopy, screening with colonoscopy, ${ }^{30}$ and receipt of a follow-up wellness exam (including Pap test, mammogram referral, clinical breast exam, and human papillomavirus test $)^{40}$ among participants after $\mathrm{CHW} / \mathrm{PN}$ interventions.

Among studies in this review that specifically targeted under-screened individuals $\left(n=10\right.$; RCT, ${ }^{31,32,35-}$ 37,46 quasi-experimental trial, ${ }^{33,45}$ pre-post comparison, ${ }^{48}$ prospective single arm cohort $^{27}$ ), screening rates improved for women overdue for a mammogram or never screened, ${ }^{36,37,45,48}$ women at risk for inadequate Pap test screening and follow-up, ${ }^{27}$ and men and women noncompliant with colorectal cancer screening guidelines ${ }^{31-33,35}$ after participating in $\mathrm{CHW} / \mathrm{PN}$ interventions. Additionally, among women who missed a follow-up diagnostic appointment for breast cancer, a significant proportion completed their diagnostic procedures after $\mathrm{CHW} / \mathrm{PN}$ intervention. ${ }^{46}$

Among studies in this review that specifically intervened among individuals with abnormal screening results ( $n=3$, all quasi-experimental trial), women with an abnormal breast or cervical screening result had a significantly shorter time to diagnosis than controls for cervical cancer screening ${ }^{26}$ after 30 days $^{34}$ and for breast screening ${ }^{34}$ if resolved after 60 days exposed to a $\mathrm{CHW} / \mathrm{PN}$ intervention. ${ }^{26}$ Individuals with a cancer diagnosis or abnormality and who participated in a $\mathrm{CHW} / \mathrm{PN}$ intervention had significantly higher odds of obtaining a definitive diagnosis within 1 year compared with the control group. ${ }^{49}$

\section{CHW/PN intervention characteristics}

Activities conducted, methods of communication with client, and documentation of activities. Table 3 provides details on activities conducted by $\mathrm{CHW} / \mathrm{PNs}$, including how they communicated with their clients and documented their activities. Common intervention 
Table 3. Activities Conducted by the Community Health Worker/Patient Navigator, Communication Method with Clients, and Documentation of Activities

\begin{tabular}{|c|c|c|}
\hline Activities $^{\mathrm{a}}$ & $n$ & References \\
\hline Provide education and awareness & 13 & $29,32,33,35,38-44,48,49$ \\
\hline Identify and address barriers to care & 10 & $26,30,34,36,37,40,41,42,45,48$ \\
\hline Schedule appointments & 9 & $31,32,35,36,39,40,45,47,48$ \\
\hline Attend appointments & 8 & $27,28,32,35,45-48$ \\
\hline Provide or facilitate referrals to medical care and/or support services & 7 & $27,28,30,36-45$ \\
\hline Provide appointment reminders & 6 & $30,31,35,46-48$ \\
\hline Provide motivational support and encouragement & 6 & $32,33,37,46,48,49$ \\
\hline Provide information on procedure and/or appointment preparation & 5 & $30,32,35,45,46$ \\
\hline Arrange or provide transportation & 5 & $35,36,38,47,48$ \\
\hline Identify patients due for screening & 4 & $27,28,30,34$ \\
\hline Identify medical and social risks & 3 & $27,28,37$ \\
\hline Enroll in insurance/address insurance issues & 3 & $32,48,47$ \\
\hline Monitor follow-up through resolution & 3 & $30,34,49$ \\
\hline Facilitate communication with providers & 2 & 45,49 \\
\hline Conduct community outreach & 2 & 38,49 \\
\hline \multicolumn{3}{|l|}{ Communication Mode ${ }^{\mathrm{b}}$} \\
\hline Telephone, in person & 9 & $32,34,35,39,45-49$ \\
\hline In person only & 8 & $27-29,36,38,40,43,44$ \\
\hline Telephone, mail, in person & 3 & $26,41,42$ \\
\hline Telephone only & 3 & $31,33,37$ \\
\hline Not discussed & 1 & 30 \\
\hline \multicolumn{3}{|l|}{ Documentation of activities ${ }^{a}$} \\
\hline Documentation discussed & 10 & $26,30,32,33,35,39,41,45,46,49$ \\
\hline Electronic medical record & 5 & $26,30,32,35,49$ \\
\hline Paper & 1 & 33 \\
\hline Format not specified & 4 & $39,41,45,46$ \\
\hline Documentation not discussed & 14 & $27-29,31,34,36-38,40,42-44,48$ \\
\hline
\end{tabular}

${ }^{\mathrm{a}}$ Articles may be included in more than one category.

${ }^{\mathrm{b}}$ Categories are mutually exclusive.

activities included providing education and increasing awareness, ${ }^{29,32,33,35,38-44,48,49}$ identifying and addressing barriers, ${ }^{26,30,34,36,37,40-42,45,48}$ assisting with scheduling appointments, ${ }^{31,32,35,36,39,40,45,47,48}$ and attending appointments with clients. ${ }^{27,28,32,35,45-48}$ Clinic-based CHW/PNs most commonly identified and addressed barriers to care and/or provided referrals for medical and social services, while community-based $\mathrm{CHW} /$ PNs more often focused on cancer education and awareness. Most CHW/PNs communicated with clients both in-person and by telephone. . $^{32,34,35,39,45-49}$ Three interventions were solely telephone-based. ${ }^{31,33,37}$ Five studies, all of which involved clinic-based interventions, reported that CHW/PNs had access to patient electronic health records (EHRs) and documented their activities and client interactions in the EHR. ${ }^{26,30,32,35,49}$ Other studies noted that CHW/PNs maintained paper records of their activities, ${ }^{33}$ and other practices that were not detailed in the articles. ${ }^{39,41,45,46}$

Training and educational background of CHW/PNs. Table 4 outlines the topics covered by trainings that $\mathrm{CHW} / \mathrm{PNs}$ received over the course of the studies and their educational/professional background. Most often, training provided to $\mathrm{CHW} / \mathrm{PNs}$ included general information related to cancer and health, ${ }^{27-29,32,33,38,41,42,46-48}$ cancer screening and guidelines, ${ }^{27-29,32,33,35,38,45,46,48} \mathrm{pa}$ tient support and care ${ }^{36,45,47,48}$ and overview of the project/study. ${ }^{40-42,46}$ Skill-based training on topics such as motivational interviewing, ${ }^{32,33,35,48}$ and communication $^{32,33,36,37}$ were also provided to CHW/PNs.

CHW/PNs usually had a high school education, with or without some healthcare experience, ${ }^{26,34,47,48}$ or were college graduates. ${ }^{33-35,42,44,46-48}$ A few CHW/ PNs were certified nurse assistants ${ }^{32,33}$ or functioned as the study research assistant. ${ }^{31}$ Some CHW/PNs had previous experience with community breast and cervical education, ${ }^{40,44}$ or were community partners or community members. ${ }^{32,33,38,43,44}$

CHW/PN supervision, medical team integration, compensation, and work designation. Table 5 describes the work environment of CHW/PNs. In these studies, $\mathrm{CHW} / \mathrm{PNs}$ were more often supervised by the research project manager or study investigator ${ }^{32-35,37,46,48}$ than by health center administrative staff, ${ }^{26,44}$ social workers, ${ }^{34}$ or outreach coordinators/specialists. ${ }^{38}$ Many CHW/PNs communicated directly with providers, ${ }^{27,28,32,33}$ and 
Table 4. Training Received by Community Health Worker/Patient Navigators Participating in the Intervention Studies, According to Location of Intervention

\begin{tabular}{lrr}
\hline Training content $^{\text {a }}$ & Number of studies & \multicolumn{1}{c}{ Study references } \\
\hline General cancer/health & 11 & $32,33,29,38,41,42,48,47-46$ \\
General screening/guidelines & 10 & $27,28,32,33,38,45,46,48$ \\
Patient support care & 4 & $36,45,47,48$ \\
Communication skills & $32,33,36,37$ \\
Motivational interviewing & $32-35,48$ \\
Project information & 4 & $40-42,46$ \\
Diagnosis/treatment & 4 & $45,46,48$ \\
Assessing and overcoming barriers & 3 & $35,36,47$ \\
Screening programs/resources & 3 & $27,38,46$ \\
Computer skills and administrative procedures & 3 & $41,42,45$ \\
Culturally appropriate care & 3 & 27,28 \\
Routine core competency assessments (content not described) & 2 & 26 \\
Providing referrals & 1 & 36 \\
Confidentiality & 1 & 45 \\
Not discussed & 1 & 7 \\
Educational/professional background ${ }^{a}$ & & 35 \\
College graduate & 3 & $30,31,34,39,43,44,49$ \\
Community partners, community members & 5 & $33-35,42,44,47-48$ \\
High school graduate (with or without healthcare experience) & $32,33,38,43,44$ \\
Experience in community breast and cervical education & $26,34,47,48$ \\
Certified nurse assistant & 40,44 \\
Research assistant & 2 & 32,33 \\
Not discussed & 2 & 31 \\
\hline
\end{tabular}

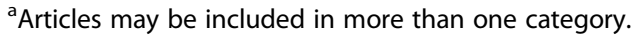

were integrated with medical teams. ${ }^{26,30,49}$ Among studies that included information on $\mathrm{CHW} / \mathrm{PNs}$ compensation, almost all reported that $\mathrm{CHW} / \mathrm{PNs}$ were paid. $^{26,28,30,31,35,36,38,42,44,47,49}$ One study used one paid and one volunteer $\mathrm{CHW} / \mathrm{PN}^{38}$ Only two articles

Table 5. Community Health Worker/Patient Navigator Supervision, Medical Team Integration, Compensation, and Work Designation

\begin{tabular}{|c|c|c|}
\hline & $n$ & References \\
\hline \multicolumn{3}{|l|}{ Supervision $^{\mathrm{a}}$} \\
\hline $\begin{array}{l}\text { Research project manager/ } \\
\text { investigator }\end{array}$ & 7 & $32-35,37,46,48$ \\
\hline Health center administrative staff & 2 & 26,44 \\
\hline Social worker navigator & 1 & \\
\hline Outreach coordinator/specialist & 1 & 38 \\
\hline Title not specified & 2 & 41,42 \\
\hline Not discussed & 12 & $27-31,36,39,40,43,45,47,49$ \\
\hline \multicolumn{3}{|l|}{ Medical team integration ${ }^{a}$} \\
\hline Communicate directly with providers & 4 & $27,28,32,33$ \\
\hline Integrated into health center team & 3 & $26,30,49$ \\
\hline Limited contact with clinicians & 1 & 35 \\
\hline Not discussed & 16 & $29,31,34,36-48$ \\
\hline \multicolumn{3}{|l|}{ Compensation $^{a}$} \\
\hline Paid & 11 & $26,28,30,31,35,36,38,42,44,47,49$ \\
\hline Volunteer & 1 & 38 \\
\hline Not discussed & 13 & $27,29,32,33,34,37,39-41,43,45,46,48$ \\
\hline \multicolumn{3}{|l|}{ Designation $^{\mathrm{b}}$} \\
\hline Part-time & 1 & \\
\hline Full-time and part-time & 1 & \\
\hline Not discussed & 22 & $26-34,36-45,48,49$ \\
\hline
\end{tabular}

${ }^{a}$ Articles may be included in more than one category.

${ }^{\mathrm{b}}$ Categories are mutually exclusive. addressed whether $\mathrm{CWH} / \mathrm{PN}$ were full- or part-time. One used full-time CHW/PNs, ${ }^{47}$ and another described utilizing both full- and part-time navigators. ${ }^{35}$

\section{Discussion}

Both the FQHC system and CHW/PNs were borne from the need to address persistent, complex health disparities among medically underserved communities. For decades, community health centers and CHW/PNs have provided culturally competent, comprehensive health services, education, social support, and client advocacy. CHW/PNs and FQHCs have independently been recognized as essential to achieving national public health priorities to address health disparities within a changing healthcare environment. Further, there is a growing recognition of the importance of primary care and public health collaborations in reducing health disparities. $^{50}$ Understanding how linkages between FQHCs and tertiary care centers with screening, treatment, and diagnostic capabilities are developed and maintained is crucial, as is learning more about scalability and replication of effective CHW/PN interventions, and chronic disease versus disease-specific models of CHW/PN programs.

This systematic literature review sought to identify and describe CHW/PN initiatives in FQHCs related to cancer prevention and control according to key 
intervention components commonly used to measure public health institutional and programmatic capacity and sustainability. ${ }^{14,23,51-55}$ In doing so, we found that $\mathrm{CHW} / \mathrm{PN}$ programs can improve completion and timeliness of breast, cervical, and colorectal cancer screening and diagnosis among the medically underserved populations served by FQHCs. The outcomes in these studies clearly illustrate that FQHC-CHWs/ PNs partnerships can serve the unique needs of diverse and underserved communities in both clinical and community settings.

The studies included in this review were largely supported through federal funding and led by academic institutions. The controlled research environment allows investigators to examine the impact of $\mathrm{CHW} / \mathrm{PN}$ interventions on screening and diagnostic outcomes in FQHC patients, but these controlled environments often do not reflect the strained reality of real-world $\mathrm{CHW} / \mathrm{PN}$ programs where resources, training, supervision, and support are often less robust than in federally funded studies. A significant programmatic barrier to the integration of CHWs/PNs into most FQHCs is that $\mathrm{CHW} / \mathrm{PN}$ services are not billable or reimbursable. $^{56,57}$ Safety net institutions, like FQHCs, which serve populations with limited resources to support themselves and their families when cancer is diagnosed, are unlikely to have sufficient resources for $\mathrm{CHW} / \mathrm{PN}$ programs and interventions (although it has occurred). On the contrary, health systems targeting and caring for insured, employed, and educated patients are more likely to have cancer patient navigation programs. ${ }^{58}$ Ongoing cost-analysis studies and healthcare utilization studies of chronic disease $\mathrm{CHW} / \mathrm{PN}$ programs are critical, ${ }^{59}$ have documented costs and healthcare savings when CHWs are utilized, and may provide evidence of the value of sustainable funding for these services in clinical settings serving the medically underserved. ${ }^{60-62}$

In addition to sustainable funding strategies, inconsistent training and nonuniform competency standards have been recognized as barriers to broader integration of $\mathrm{CHW} / \mathrm{PNs}$ into public health programs. ${ }^{14,51}$ This study found that training content and educational/professional background varied for each of these studies, and that aspects of occupational regulation critical to establishing credibility and scope of practice, such $\mathrm{CHW} / \mathrm{PN}$ supervision, integration the into the medical team, paid or volunteer status, and part-time or full-time status, were not as often discussed.
Noted barriers and facilitators to FQHC-affiliated CHW/PN programs

Barriers reported in the literature should be considered when conducting future intervention research. For patients, the inability to take time off work to attend screening, lack of reliable transportation and childcare, and cost of copayments ${ }^{43}$; housing concerns and instability $^{27,32,33}$; and migrant or immigrant status ${ }^{32,33}$ were noted as impediments to participating in screening. Barriers faced by programs and health centers that may impact program outcomes include the inability to retain skilled bilingual $\mathrm{PNs}^{47}$; lack of onsite mammography ${ }^{27}$; framing a navigation program for "cancer patients"49; and the paper records and the inability to track screening tests conducted outside the clinic. ${ }^{49}$

Several facilitators to implementation of CHW/PN interventions in FQHCs were reported, and include partnering with churches when working with Hispanic communities, ${ }^{43}$ ensuring a common language is spoken between $\mathrm{CHWs} / \mathrm{PNs}$ and their clients, ${ }^{48}$ ensuring open communication between clinicians and PNs working in the clinic, ${ }^{32,35}$ incorporating Community Guiderecommended strategies into the $\mathrm{CHW} / \mathrm{PN}$ intervention, ${ }^{32}$ allowing $\mathrm{CHW} / \mathrm{PNs}$ flexible work hours and scheduling, ${ }^{32}$ supporting initial and ongoing training, ${ }^{26}$ allowing CHW/PN access to EHRs, ${ }^{30}$ providing reducedcost screening, ${ }^{35}$ promoting multiple types of screening exams at one time, ${ }^{40}$ incorporating a community advisory panel into CHW/PN program activities, ${ }^{26}$ and facilitating access follow-up care through National Breast and Cervical Cancer Early Detection Program (NBCCEDP). ${ }^{34}$

\section{Policy implications}

Numerous policy initiatives light the path forward for the integration of $\mathrm{CHW} / \mathrm{PNs}$ into primary care and public health. Effective January 2014, CMS created a final rule (CMS-2334-F) that opens up payment opportunities for preventive services by nonlicensed individuals (e.g., CHWs) recommended by physicians or other licensed practitioners of the health arts. ${ }^{63}$ The rule change presents an important opportunity for enhancing the focus on prevention through the Medicaid program. ${ }^{64}$ ACA also offers state Medicaid programs the opportunity to create Health Homes for beneficiaries living with chronic illness, and several states have Medicaid state plan amendments that explicitly include or refer to $\mathrm{CHWs}{ }^{59,64-66}$ Finally, ACA creates funding for state innovation models (SIM) designed to support states in the development and testing of state-based models for multi-payer payment and healthcare 
delivery system transformation. ${ }^{67}$ Of six states currently implementing their SIM designs, four have included CHWs in their plans. In Round One of the SIM initiative, nearly $\$ 300$ million was awarded to 25 states to design or test improvements to their public and private health payment and delivery systems (https:// innovation.cms.gov/initiatives/state-innovations/). ${ }^{64}$

Another opportunity to support integration of $\mathrm{CHWs} /$ PNs into FQHCs and other community-based primary care setting is the patient-centered medical home model. ${ }^{68-70}$ The patient-centered medical home is a way of organizing primary care that emphasizes care coordination and communication, treatment of the many needs of patients at the same time, increased access to care, and empowerment of patients to be partners in their own care. The development of a $\mathrm{CHW} / \mathrm{PN}$ program in a health center may assist in meeting the requirements for patient-centered medical home designation. ${ }^{71}$

Strengths and limitations. To our knowledge, there has been no assessment of $\mathrm{CHW}$ and $\mathrm{PN}$ activities in FQHCs on a national, state, or local scale. The results of this systematic literature review provide an overview regarding cancer prevention and diagnostic $\mathrm{CHW} / \mathrm{PN}$ programs in FQHCs targeting the medically underserved that can be used to design future interventions. We note several limitations to our systematic literature review methods and results. Studies were limited to those published in the peer-reviewed literature. This may have limited study to those conducted by or in partnership with academic researchers. The peerreview literature does not capture all $\mathrm{CHW} / \mathrm{PN}$ activities ongoing at FQHCs as programs may not have the capacity to effectively evaluate and publish their work, and FQHC-designation may not have been included in the study site description. The studies included in this review varied in design and methodology (RCTs, quasiexperimental trials, and pre-post comparison) and the impact of those variations to study outcomes was not assessed, but should be acknowledged when interpreting the results of this literature review. Additionally, the reporting period for this review ended in December 2013, potentially excluding additional literature relevant to the search.

\section{Conclusion}

Better partnering and integration of public health and primary care interventions and systems can change the context within which chronic disease occurs and reduce health disparities. As evident in this review, $\mathrm{CHW} / \mathrm{PN}$ interventions implemented in FQHCs or coordinated with FQHCs are effective in increasing cancer screening and the timeliness of diagnostic resolution among medically underserved populations. Bridging the community-clinical divide is critical to supporting disadvantaged communities to gain access to primary care, including cancer screening. Although evidence supports engaging $\mathrm{CHW} / \mathrm{PNs}$ in the national health delivery system, lack of sustainable resources for these programs, including reimbursement for services, remains a challenge. Local, regional, and state programs will likely need to continue innovating and collaborating to develop and sustain systems and programs for the medically underserved in their communities. Looking forward, national public health agencies must continue to promote the development and adoption of evidence-based interventions and strategies that will reduce health disparities. Achieving health equity in cancer and other chronic diseases is a real and pressing priority for public health, and fostering collaboration between $\mathrm{CHWs} / \mathrm{PN}$ programs and FQHCs and other community-based settings is potentially a very powerful tool to achieve public health goals.

\section{Acknowledgment}

Centers for Disease Control and Prevention, Contract \#200-2013-M-57606 to NOVA Research Company, Inc.

\section{Author Disclosure Statement}

There are no conflicts of interest to report, or financial disclosures. Preparation of this article was entirely funded by the United States Government. All authors are federal government employees or contractors and this report not subject to copyright in the United States. The findings and conclusions in this report are those of the authors and do not necessarily represent the official position of the Centers for Disease Control and Prevention.

\section{References}

1. Centers for Disease Control and Prevention. Cancer screening-United States, 2010. MMWR Morb Mortal Wkly Rep. 2012;61:41-45.

2. Ward E, Jemal A, Cokkinides V, et al. Cancer disparities by race/ethnicity and socioeconomic status. CA: Cancer J Clin. 2004;54:78-93.

3. Dean HD, Williams KM, Fenton KA. From theory to action: applying social determinants of health to public health practice. Public Health Rep. 2013;128 Suppl 3:1-4.

4. Frieden TR. A framework for public health action: the health impact pyramid. Am J Public Health. 2010;100:590-595.

5. Marmot M, Friel S, Bell R, et al. Closing the gap in a generation: health equity through action on the social determinants of health. Lancet. 2008;372:1661-1669.

6. Bauer UE, Briss PA, Goodman RA, et al. Prevention of chronic disease in the 21st century: elimination of the leading preventable causes of premature death and disability in the USA. Lancet. 2014;384:45-52. 
7. Frieden TR, Myers JE, Krauskopf MS, et al. A public health approach to winning the war against cancer. Oncologist. 2008;13:1306-1313.

8. National Prevention Council. National Prevention Strategy. Washington, DC: U.S. Department of Health and Human Services, Office of the Surgeon General, 2011.

9. Kim K, Choi JS, Choi E, et al. Effects of community-based health worker interventions to improve chronic disease management and care among vulnerable populations: a systematic review. Am J Public Health. 2016;106:e3-e28.

10. Sabatino SA, Lawrence B, Elder R, et al. Effectiveness of interventions to increase screening for breast, cervical, and colorectal cancers: nine updated systematic reviews for the guide to community preventive services. Am J Prev Med. 2012;43:97-118.

11. American Public Health Association. Support for Community Health Workers to Increase Health Access and to Reduce Health Inequities. Policy Statement No. 20091. 2009.

12. Perez LM, Martinez J. Community health workers: social justice and policy advocates for community health and well-being. Am J Public Health. 2008;98:11-14.

13. Freeman HP, Rodriguez RL. History and principles of patient navigation. Cancer. 2011;117:3539-3542.

14. Improving cancer prevention and control: how state health agencies can support patient navigators and community health workers. The Association of State and Territorial Health Officials, 2012. Available at http:// www.astho.org/ImprovingCancerPreventionandControl/ Accessed May 23, 2017

15. Wells KJ, Battaglia TA, Dudley DJ, et al. Patient navigation: state of the art or is it science? Cancer. 2008;113:1999-2010.

16. Wells KJ, Luque JS, Miladinovic B, et al. Do community health worker interventions improve rates of screening mammography in the United States? A systematic review. Cancer Epidemiol Biomarkers Prev. 2011;20:1580-1598.

17. HRSA Bureau of Primary Care. Health center program: impact and growth 2016. Available at https://bphc.hrsa.gov/about/healthcenterprogram/ index.html Accessed May 23, 2017.

18. Adams SA, Choi SK, Khang L, et al. Decreased cancer mortality-toincidence ratios with increased accessibility of federally qualified health centers. J Community Health. 2015;40:633-641.

19. Allen $C L$, Harris JR, Hannon PA, et al. Opportunities for improving cancer prevention at federally qualified health centers. J Cancer Educ. 2013;29:30-37.

20. The Patient Protection and Affordable Care Act, Pub L No. 111-148, 124 Stat. 1004 (March 2010) as amended by the Health Care and Education Reconciliation Act of 2010, Pub L No. 111-152, 124 Stat. 1083 (March 2010)

21. Shin P, Rosenbaum S, Paradise J. Community Health Centers: The Challenge of Growing to Meet the Need for Primary Care in Medically Underserved Communities. Edited by The Henry J. Kaiser Family Foundation. Washington DC, 2012. Available at http://www.nhchc.org/wp-content/ uploads/2011/09/Kaiser-health-center-challenges-March-2012.pdf Accessed May 23, 2017

22. Axelrod DA, Millman D, Abecassis MM. US Health Care Reform and Transplantation, Part II: impact on the public sector and novel health care delivery systems. Am J Transplant. 2010;10:2203-2207.

23. DeGroff A, Coa K, Morrissey KG, et al. Key considerations in designing a patient navigation program for colorectal cancer screening. Health Promot Pract. 2013;15:483-495.

24. Paskett ED, Harrop JP, Wells KJ. Patient navigation: an update on the state of the science. CA Cancer J Clin. 2011;61:237-249.

25. Viswanathan $M$, Kraschnewski JL, Nishikawa B, et al. Outcomes of Community Health Worker Interventions. Edited by R. T. I. InternationalUniversity of North Carolina Evidence-based Practice Center. 2009. Available at www.ahrq.gov/research/findings/evidence-based-reports/ comhwork-evidence-report.pdf Accessed May 23, 2017.

26. Battaglia TA, Bak SM, Heeren T, et al. Boston patient navigation research program: the impact of navigation on time to diagnostic resolution after abnormal cancer screening. Cancer Epidemiol Biomarkers Prev. 2012;21:1645-1654.

27. Clark CR, Baril N, Hall A, et al. Case management intervention in cervical cancer prevention: the Boston REACH coalition women's health demonstration project. Prog Community Health Partnersh. 2011;5:235-247.
28. Clark CR, Baril N, Kunicki M, et al.; REACH 2010 Breast and Cervical Cancer Coalition. Addressing social determinants of health to improve access to early breast cancer detection: results of the Boston REACH 2010 Breast and Cervical Cancer Coalition Women's Health Demonstration Project. J Womens Health (Larchmt). 2009;18:677-690.

29. Gotay CC, Banner RO, Matsunaga DS, et al. Impact of a culturally appropriate intervention on breast and cervical screening among native $\mathrm{Ha}-$ waiian women. Prev Med. 2000;31:529-537.

30. Honeycutt S, Green R, Ballard D, et al. Evaluation of a patient navigation program to promote colorectal cancer screening in rural Georgia, USA. Cancer. 2013;119:3059-3066.

31. Jandorf L, Gutierrez Y, Lopez J, et al. Use of a patient navigator to increase colorectal cancer screening in an urban neighborhood health clinic. J Urban Health. 2005;82:216-224.

32. Lasser KE, Murillo J, Lisboa $\mathrm{S}$, et al. Colorectal cancer screening among ethnically diverse, low-income patients: a randomized controlled trial. Arch Intern Med. 2011;171:906-912.

33. Lasser KE, Murillo J, Medlin E, et al. A multilevel intervention to promote colorectal cancer screening among community health center patients: results of a pilot study. BMC Fam Pract. 2009;10:37.

34. Markossian TW, Darnell JS, Calhoun EA. Follow-up and timeliness after an abnormal cancer screening among underserved, urban women in a patient navigation program. Cancer Epidemiol Biomarkers Prev. 2012;21:1691-1700.

35. Percac-Lima S, Grant RW, Green AR, et al. A culturally tailored navigator program for colorectal cancer screening in a community health center: a randomized, controlled trial. J Gen Intern Med. 2009;24:211-217.

36. Russell KM, Champion VL, Monahan PO, et al. Randomized trial of a lay health advisor and computer intervention to increase mammography screening in African American women. Cancer Epidemiol Biomarkers Prev. 2010;19:201-210.

37. West DS, Greene P, Pulley L, et al. Stepped-care, community clinic interventions to promote mammography use among low-income rural African American women. Health Educ Behav. 2004;31:29S-44S.

38. Earp JA, Eng E, O'Malley MS, et al. Increasing use of mammography among older, rural African American women: results from a community trial. Am J Public Health. 2002;92:646-654.

39. Fernandez ME, Gonzales A, Tortolero-Luna G, et al. Effectiveness of Cultivando la Salud: a breast and cervical cancer screening promotion program for low-income Hispanic women. Am J Public Health. 2009;99:936943.

40. Hunter JB, de Zapien JG, Papenfuss M, et al. The impact of a promotora on increasing routine chronic disease prevention among women aged 40 and older at the U.S.-Mexico border. Health Educ Behav. 2004;31:18S-28S

41. Katz ML, Tatum CM, Degraffinreid CR, et al. Do cervical cancer screening rates increase in association with an intervention designed to increase mammography usage? J Womens Health (Larchmt). 2007;16:24-35.

42. Paskett E, Tatum C, Rushing J, et al. Randomized trial of an intervention to improve mammography utilization among a triracial rural population of women. J Natl Cancer Inst. 2006;98:1226-1237.

43. Sauaia A, Min SJ, Lack D, et al. Church-based breast cancer screening education: impact of two approaches on Latinas enrolled in public and private health insurance plans. Prev Chronic Dis. 2007;4:A99.

44. Welsh AL, Sauaia A, Jacobellis J, et al. The effect of two church-based interventions on breast cancer screening rates among Medicaid-insured Latinas. Prev Chronic Dis. 2005;2:A07.

45. Burhansstipanov L, Dignan MB, Schumacher A, et al. Breast screening navigator programs within three settings that assist underserved women. J Cancer Educ. 2010;25:247-252.

46. Maxwell $A E$, Jo $A M$, Crespi $C M$, et al. Peer navigation improves diagnostic follow-up after breast cancer screening among Korean American women: results of a randomized trial. Cancer Causes Control. 2010;21:1931-1940.

47. Percac-Lima S, Ashburner JM, Bond B, et al. Decreasing disparities in breast cancer screening in refugee women using culturally tailored patient navigation. J Gen Intern Med. 2013;28:1463-1468.

48. Percac-Lima S, Milosavljevic B, Oo SA, et al. Patient navigation to improve breast cancer screening in Bosnian refugees and immigrants. J Immigr Minor Health. 2012;14:727-730.

49. Warren-Mears V, Dankovchik J, Patil M, et al. Impact of patient navigation on cancer diagnostic resolution among Northwest Tribal communities. J Cancer Educ. 2013;28:109-118. 
50. Institute of Medicine. Primary Care and Public Health Exploring Integration to Improve Population Health. Washington, DC: The National Academies Press, 2012.

51. Addressing chronic disease through community health workers: a policy and systems-level approach. Centers for Disease Control and Prevention; Division for Health Disease and Stroke Prevention, 2015. Available at https://www.cdc.gov/dhdsp/docs/chw_brief.pdf Accessed May 23, 2017.

52. Frieden TR. Six components necessary for effective public health program implementation. Am J Public Health. 2014;104:17-22.

53. Meyer AM, Davis M, Mays GP. Defining organizational capacity for public health services and systems research. J Public Health Manag Pract. 2012;18:535-544.

54. Scheirer MA, Dearing JW. An agenda for research on the sustainability of public health programs. Am J Public Health. 2011;101:2059-2067.

55. Schell SF, Luke DA, Schooley MW, et al. Public health program capacity for sustainability: a new framework. Implement Sci. 2013;8:15.

56. Brownstein JN, Bone LR, Dennison CR, et al. Community health workers as interventionists in the prevention and control of heart disease and stroke. Am J Prev Med. 2005;29:128-133.

57. Trust for America's Health and Nemours. Medicaid reimbursement for community-based prevention. Available at www.astho.org/CommunityHealth-Workers/Medicaid-Reimbursement-for-Community-BasedPrevention/ Accessed May 23, 2017.

58. Freund KM. Patient navigation: the promise to reduce health disparities. $J$ Gen Intern Med. 2011;26:110-112.

59. Shah MK, Heisler M, Davis MM. Community health workers and the Patient Protection and Affordable Care Act: an opportunity for a research, advocacy, and policy agenda. J Health Care Poor Underserved. 2014;25:17-24.

60. Fedder DO, Chang RJ, Curry S, et al. The effectiveness of a community health worker outreach program on healthcare utilization of west Baltimore City Medicaid patients with diabetes, with or without hypertension. Ethn Dis. 2003;13:22-27.

61. Mirambeau AM, Wang G, Ruggles L, et al. A cost analysis of a community health worker program in rural Vermont. J Community Health. 2013;38:1050-1057.

62. Prezio EA, Pagan JA, Shuval K, et al. The Community Diabetes Education (CoDE) program: cost-effectiveness and health outcomes. Am J Prevent Med. 2014;47:771-779.

63. Department of Health and Human Services. Update on preventive services initiative. 2013. Available at https://www.medicaid.gov/federalpolicy-guidance/downloads/cib-11-27-2013-prevention.pdf Accessed May 23, 2017.

64. Katzen A, Morgan M. Affordable Care Act Opportunities for Community Health Workers. Boston, MA: Harvard Law School Center for Health Law and Policy, 2014.
65. The Patient Protection and Affordable Care Act. Pub L No. 111148, 124 Stat 119; Title II: Subtitle I, $§ 2703$ (March 2010); Centers for Medicare \& Medicaid Services. Approved Health Home State Plan Amendments. Baltimore, MD. Accessed December 2, 2016. Available at www.medicaid.gov/State-Resource-Center/MedicaidState-Technical-Assistance/Health-Homes-Technical-Assistance/ Approved-Health-Home-State-Plan-Amendments.html Accessed May 23, 2017.

66. Islam N, Nadkarni SK, Zahn D, et al. Integrating community health workers within Patient Protection and Affordable Care Act implementation. J Public Health Manag Pract. 2015;21:42-50.

67. The Patient Protection and Affordable Care Act. Pub L No. 111-148, 124 Stat 119; Title III: Subtitle A, $\S 3021$ (March 2010).

68. Johnson SL, Gunn VL. Community health workers as a component of the health care team. Pediatr Clin North Am. 2015;62:1313-1328.

69. Sarfaty $M$, Wender $R$, Smith R. Promoting cancer screening within the patient centered medical home. CA: Cancer J Clin. 2011;61:397408.

70. Taylor EF, Machta RM, Meyers DS, et al. Enhancing the primary care team to provide redesigned care: the roles of practice facilitators and care managers. Ann Fam Med. 2013;11:80-83.

71. Martinez J, Ro M, Villa NW, et al. Transforming the delivery of care in the posthealth reform era: what role will community health workers play? Am J Public Health. 2011;101:e1-e5.

Cite this article as: Roland $\mathrm{KB}$, Milliken $\mathrm{EL}$, Rohan $\mathrm{EA}$, DeGroff $\mathrm{A}$ White S, Melillo S, Rorie WE, Signes CC, Young PA (2017) Use of community health workers and patient navigators to improve cancer outcomes among patients served by federally qualified health centers: a systematic literature review, Health Equity 1:1, 61-76, DOI: 10.1089/heq.2017.0001.

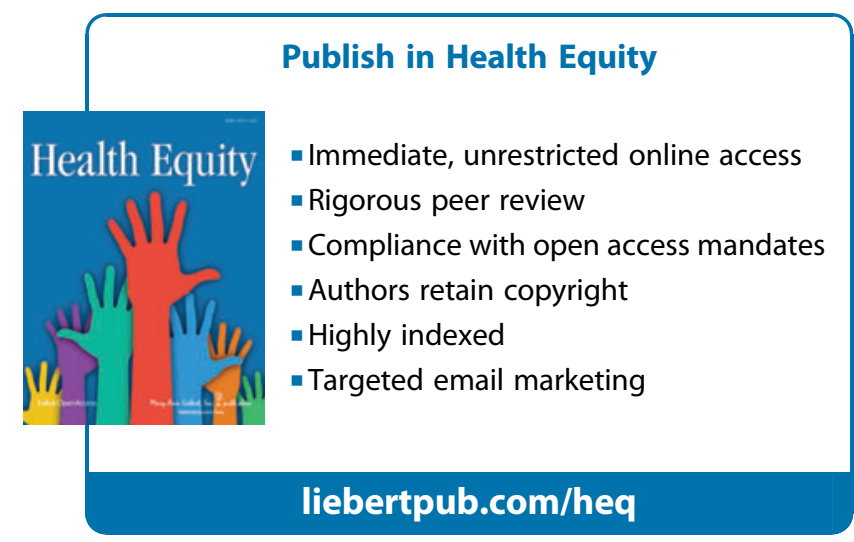

